\title{
Excitation of Waves in a Belousov-Zhabotinsky System in Emulsion Media
}

\author{
C. Basavaraja, R. Pierson, and Do Sung Huh*
}

\author{
Department of Chemistry and Institute of Functional Materials, Inje University: Krwngnam 621-749, Korea \\ E-mail: chemhds arinje.ack \\ Received September 2, 2008
}

\begin{abstract}
This study delves on the chemical wave propagation obtained by a modified Belousov-Zhabotinsky (BZ) reaction in a two-dimensional system under the AOT-enulsion media conditions. The media consist of water, oil (octane), sodium bis-sulphosuccinate as an anionic surfactant, and the reactants containing ferroin and 1,4cyclohexanedione (1,4-CHD). Results on the concentration dependence of the overall dynamics and wave speeds are presented. The behaviors obtained in this system are analyzed by a suitable mechanism specifically for their reaction systems. These behaviors are then compared with the wave pattems observed under sinilar systems adopting resin or gel membrane system.
\end{abstract}

Key Words : AOT-emulsion, BZ reaction, Excitation, Patten fomation

\section{Introduction}

Chemical waves are striking phenomena that occur in some reacting systems evolving far from equilibrium. Examples of these are pulse waves propagating in the so-called excitable media. In such media, the nonlinear reactive elements are coupled with one another locally by transport processes like diffusion. After excitation, these elements become refractory, slowly recovering their receptive state, where they can be excited again. ${ }^{2}$ The Belousov-Zhabotinsky (BZ) reaction is an ideal model for the quantitative experimental analysis of generic spatio-temporal phenomena in excitable reaction-diffusion systems. ${ }^{3,4}$ Excitation waves often lead to the emergence of intricate dynamical structures that are observed in many biological, chemical, and physical systems. ${ }^{5}$ Controlling the dynamics of an excitable system of either a reaction system or reaction medium is an important field of current research. The BZ reaction system is the most suitable experimental model for the study of wave propagation in an excitable medium. Moreover, it provides efficient control of wave dynamics and can be illustrated by a chemical procedure and physical methods such as electrical fields and light illumination. ${ }^{6}$

The aerosol water-in-oil (AOT) emulsion media is a very promising subject in the investigation of the reaction diffusion $(\mathrm{RD})$ phenomenon. The investigation of the $B Z$ reactions in interacting micro and nanodroplets, such as the reverse micelles, ${ }^{7}$ is another field of study in nonlinear chemical systems. The study of patterns in this kind of system was intended to mimic the biochemical systems such as those which contain lipids. ${ }^{8}$ Recently, Vanag et al. have studied in detail the behavior of the $\mathrm{BZ}$ reaction in water nanodroplets of a reverse emulsion. ${ }^{7 \cdot 10}$ An AOT assembly contains a small polar group and two long fat tails facing the hydrophobic phase. There is a unique way of arranging such assemblies closely together: by placing them head-to-head or tail-to-tail without intervening gaps. Specifically, their polar heads are oriented towards the center of the sphere formed by the hydrophobic tails. These geometric properties of the AOT assemblies account for its inverse character, where the water cores surrounded by a monolayer of AOT assemblies are submerged in the continuous hydrophobic phase. ${ }^{11,12}$ A study of the BZ-AOT reaction system is readily applicable to the pattern formation in the $\mathrm{RD}$ system. ${ }^{13,14}$ This study could trigger the discovery of unknown fundamental physical and chemical laws governing the behavior of nonlinear systems.

Over the years, various new substrates have been introduced to replace MA to create a bubble-free medium. In spite of the many known substrate-analogs of the classical $\mathrm{BZ}$, only a few have been utilized for studies on propagating waves so far. The rationale behind the use of new substrates is the possibility that the investigation of wave propagation in the $\mathrm{BZ}$ systems containing new kinds of organic substrates, can determine new and dynamic phenomena, which were not previously observed in the classical system. The $\mathrm{BZ}$ reaction adopting 1,4-cyclohexanedione (1,4-CHD) is identical to the classic $\mathrm{BZ}$ reaction except that $1,4-\mathrm{CHD}$ is used in lieu of MA. This new reaction system has the advantage of inducing mechanical dynamics, which will enable a long reaction time without nucleating gas bubbles in the closed system. A system containing 1,4-CHD as the organic substrate and ferroin as the catalyst was found to exhibit interesting nonlinear phenomena characterized by wave stacking and merging. ${ }^{15,16}$

In the present report, the characteristic behaviors for the pattern formation of the $\mathrm{BZ}$ reaction system in an AOT reaction media are studied. This was conducted by adopting $1,4-\mathrm{CHD}$ as an organic reagent. Reagent $1,4-\mathrm{CHD}$ is known to show an unusual dynamic pattern of traveling waves that the malonic acid system (MA) does not exhibit. The sodium bis-sulphosuccinate used as a media of AOT contains a small polar group ( $\mathrm{SO}_{3}{ }^{-}$group) and two long fat tails facing the hydrophobic phase. Specifically, their polar heads face towards the center to form a sphere. Such geometric properties of the AOT molecules can be considered if only for the 
inverse character of AOT-emulsion with water cores surrounded by a monolayer of AOT molecules submerged in the continuous hydrophobic phase (octane). Both the induction and duration times are analyzed based on the concentration of the overall dynamics and wave speeds. The duration time of the wave propagation is shortened by increasing the initial concentration of 1,4-CHD, bromate, and ferroin. By scanning through a range of chemical concentrations, different patterns based on the bifurcation behavior concentrations in the modified $\mathrm{BZ}$ system adopting 1,4-CHD were obtained. The induction period and the excitable behavior of the BZ-AOT are discussed. The behavior of the aforementioned experimental system is compared with similar experiments involving gel membrane and resin media, which have been discussed in the context of the modified BZ system.

\section{Experimental Section}

Except of ferroin (Fluka), the analytical grade reagents used in the experimental study were purchased from Sigma Aldrich. These reagents were used without further purification. The experiments were performed at room temperature of $20.0 \pm 0.5^{\circ} \mathrm{C}$. The working solutions were prepared from the stock solutions of $0.6 \mathrm{M} \times \mathrm{aBrO}, 0.2 \mathrm{M}$ 1,4-CHD. $4.5 \mathrm{M}$ sulfuric acid, and $25 \mathrm{mM}$ ferroin solution in distilled water. To study the $\mathrm{BZ}$ reaction, an emulsion medium (prepared using $1.5 \mathrm{M}$ sodium bis- $\alpha$-sulphosuccinate in octane) was placed in a Petri dish with a $3.5 \mathrm{~cm}$ diameter to obtain a media with the thickness 1-2 $\mathrm{mm}$. The liquid emulsion was added into the Petri dish and dried in an oven for about 8-10 $\mathrm{h}$ at $25^{\circ} \mathrm{C}$ to get a smooth and uniform surface. The $\mathrm{BZ}$ solutions were poured over the dried surface of the emulsion to form a uniform thickness required to generate patterns. After adding the $\mathrm{BZ}$ solution. the Petri dish was covered with a lid, which served as a window for observing the patterns. The experiments were monitored with a digital imaging system. An image from a CCD camera (Sony, SSC370 ) attached to a zoom lens (Niko, 1.4X) was processed into a computer image analysis program (Flash Point, Opimus 6.1). The wave characters including the wave velocity and wavelength were determined by the measurement of processing time in the computer. The surface plot was obtained from the figures using the software called Image. In the Figures 1 and 4, 70 pixel is a short distance/part corresponding about $0.7 \mathrm{~cm}$.

\section{Results and Discussion}

Typical patterns in the AOT system. Figure 1a-1e show typical patterns of spiral waves spotted at various times and the plot profiles under the emulsion medium conditions. The concentrations for the sequential pattern taken as the standard condition are $\left[\mathrm{BrO}_{3}^{-}\right]_{0}=0.6 \mathrm{M},[1,4-\mathrm{CHD}]_{0}=0.12 \mathrm{M}$. [ferroin $]_{0}=0.49 \mathrm{M}$, and $\left[\mathrm{H}^{+}\right]_{0}=0.3 \mathrm{M}$. The observed patterns are initially induced by the periodic bursts as shown in Figure $1 \mathrm{~b}$. The patterns gave rise to spiral waves that later (a)
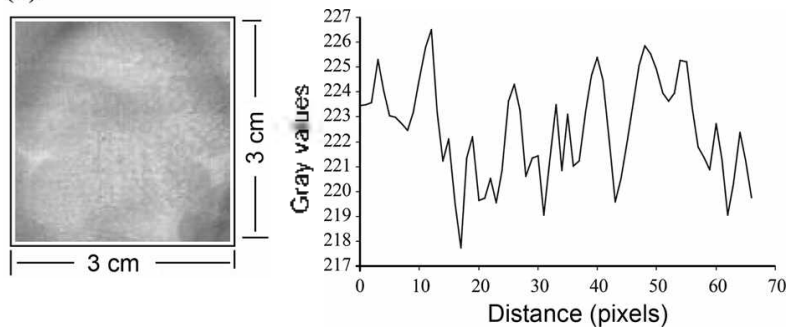

(b)
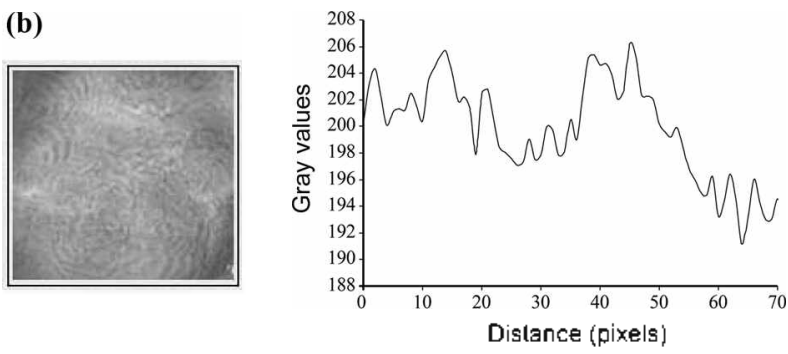

(c)
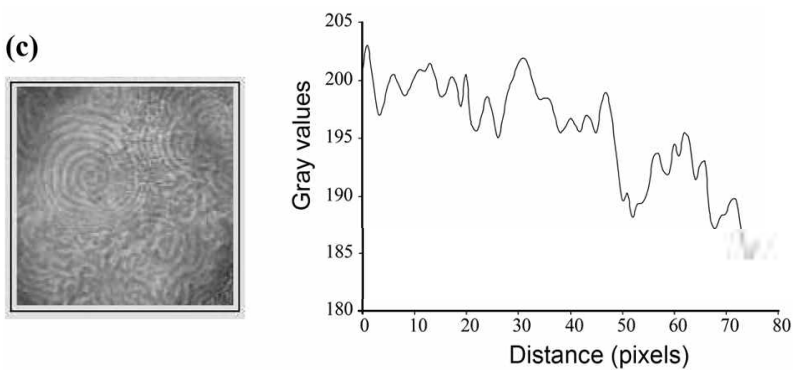

(d)
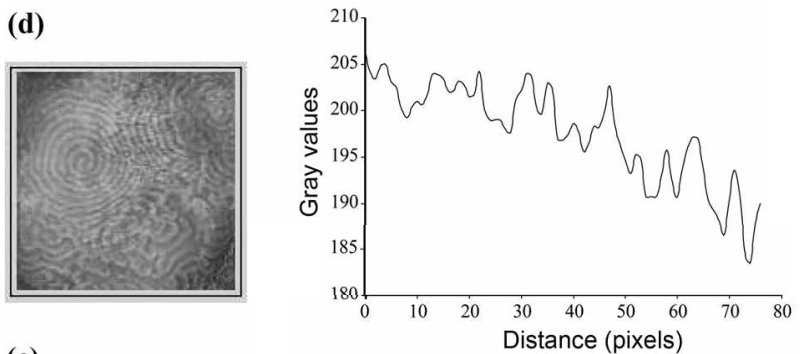

(e)
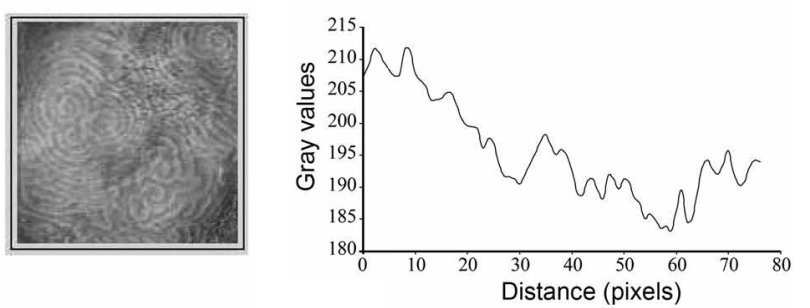

(f)
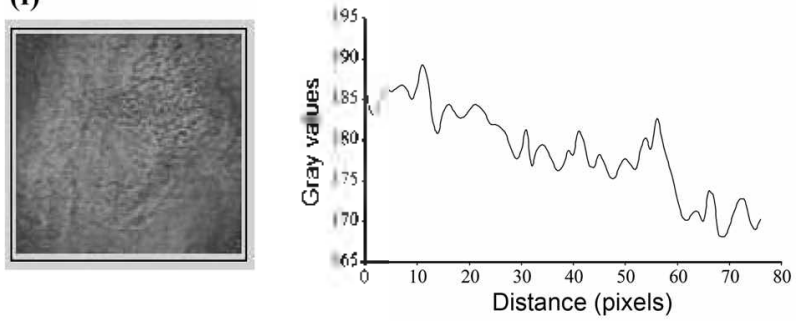

Figure 1. Typical propagating waves with a spiral pattern obtained in a micro-emulsion medium at $\left[\mathrm{BrO}_{3}^{-}\right]_{13}=0.6 \mathrm{M},[1,4-\mathrm{CHD}]_{1)}=$ 0.12 . [ferroin] $\mathrm{g}=0.49 \mathrm{mM}$. and $[\mathrm{H}] \mathrm{g}=0.3 \mathrm{M}$ : and at $t=$ (a) 30 . (b) 60 . (c) 115 . (d) 130 . (e) 136 , and (f) $170 \mathrm{~min}$. The figures in the right-hand side of the wave patterns show their plot profiles in each time. 
transformed into multicentral spiral waves. They further split in parallel spiral form with a characteristic wavelength between $1.5-2.0 \mathrm{~mm}$. Figure 1c displays the characteristic spiral waves formed at $115 \mathrm{~min}$. The waves slowly changed to form three-armed spirals at $130 \mathrm{~min}$. The new waves then split and moved apart, subsequently joining the two spirals. When a wave between two basins split into two new waves to merge with the neighboring spirals, other waves (e.g. those observed in Figures 1d, and le) moved into the vacant area perpendicular to the line joining the spiral centers. The life period for these waves was set at $-180 \mathrm{~min}$.

Excitable media are spatially distributed systems that have the ability to propagate signals without damping. An excitable media system can be considered as a group of individual elements attached to each other. Each element can pass information to its neighbors. In the physical system. signals are passed by diffusion. In our experiment, a similar behavior was observed. Reaction chemical species such as $\mathrm{HBrO}_{2}$ and $\mathrm{Fe}^{3+}$ diffuse to the neighboring regions. Each coupled point in the excitable media is characterized by a rest state characterized by stability against perturbations. This can be analyzed by a plot profile shown in Figure 1 together with its wave pattern, which displays a two-dimensional graph of the intensities of pixels along a line within the image in their $\mathrm{x}$-axis. The $\mathrm{x}$-axis represents the distance along the line and the $y$-axis represents the pixel intensity. The plot profile for each moment of wave propagation shows the excitation of the behavior and the surface complexities that occur during the pattern formation. This facilitated a deeper understanding of the whole surface structure and eventually allowed us to explore the responses to it. The process of developing a profile provides a clear overview of the surface and its complexities. As can be seen in the profiles, the reaction causes profound changes in the topography. The initial pattern produced some variations in amplitude in relation to the time period. One variation is the decrease in the length of the amplitude as time progressed. The plot profiles shows that the initial excitation immediately formed the patterns into a zigzag structure. In the latter stages, this structure slowly decayed and formed a relatively uniform surface.

Concentration dependence of the wave behavior. The concentrations of $\left[\mathrm{BrO}_{3}^{-}\right]_{0}$. $[1,4-\mathrm{CHD}]_{0}$, and [ferroin $]_{0}$ for the concentration dependence of the appearance of waves were varied from 0.12 to $1.29,0.1$ to 0.39 , and $10^{-4}$ to $10^{-3}$ $\mathrm{M}$. Figures 2 and 3 show the $\left[\mathrm{BrO}_{3}^{-}\right]_{0}-[1,4-\mathrm{CHD}]_{0}$ and $[1,4-\mathrm{CHD}]_{0}$ - [ferroin $]_{0}$ bifurcation phase diagrams, respectively. The concentrations used, except those of the two reagents, are the same with those given in Figure 2. The various types of patterns obtained are separated by different lines in each diagram. It can be seen that the changes in the initial concentrations in the bifurcation diagrams led to variations in the characteristic behavior of the pattern formation. The variation of the behavior feature attains monotonicity with the initial conditions. The figures show that the reaction medium itself does not produce patterns. Pattern formation does not occur at very low or very high concentrations.

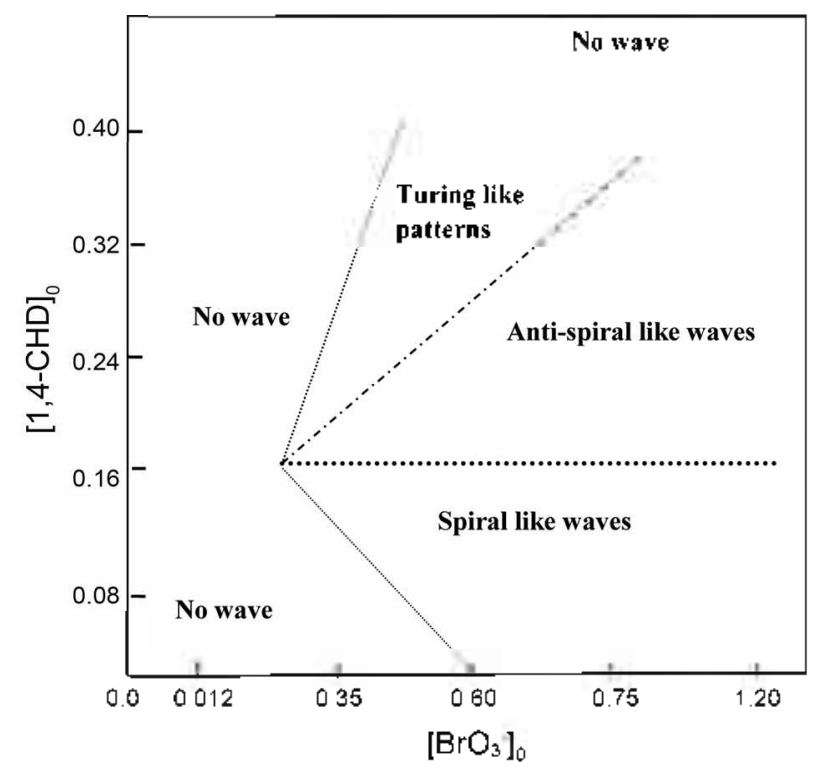

Figure 2. Bifurcation diagram showing the dependency of wave pattems to the $[1.4-\mathrm{CHD}]_{i j}-[\text { bromate }]_{i j}$ phase at $\left[\right.$ ferroin $_{i j}=0.49$ $\mathrm{mM}$ and $[\mathrm{H}]_{i j}=0.3 \mathrm{M}$.

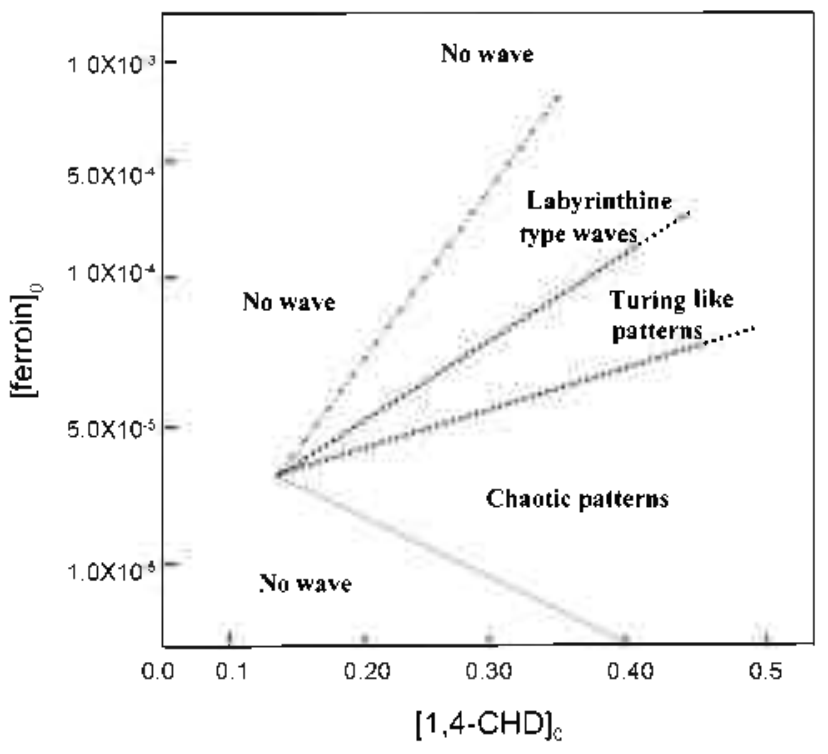

Figure 3. Bifurcation diagram showing the growth of chaotic waves and labyrinthine and hexagonal-like patterns in the $[1.4-$ $\mathrm{CHD}]_{9}-[\text { ferroin }]_{1)}$ phase at $\left[\mathrm{BrO}_{3}^{-}\right]_{9}=0.6 \mathrm{M}$ and $\left[\mathrm{H}^{-}\right]_{10}=0.3 \mathrm{M}$.

Figure 2 presents a bifurcation diagram in the $\left[\mathrm{BrO}_{3}{ }^{-}\right]_{0-}$ $[1,4-\mathrm{CHD}]_{0}$ phase. Two types of the spiral wave were formed depending on the concentration of $[1,4-\mathrm{CHD}]_{\mathrm{j}}$. At a given concentration of $\left[\mathrm{BrO}_{3}^{-}\right]_{0}$ and a low level of $[1,4-$ $\mathrm{CHD}]_{0,}$ a spiral wave that propagated from the inside to the outside was formed. A spiral-like wave is characterized with a color change. At the same $\left[\mathrm{BrO}_{3}^{-}\right]_{0}$ range, but with a high $[1,4-C H D]_{0}$ concentration, a spiral wave that propagated in a reverse direction to the inside was formed. However, at higher $[1,4-\mathrm{CHD}]_{0}$ levels, the patterns show Turing-like structures in the system. The amplitudes of the structures increased with time progress. Figure 3 illustrates a bifur- 
(a)
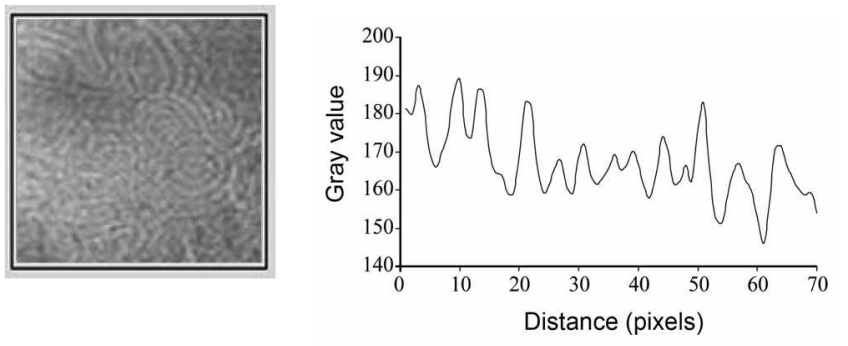

(b)

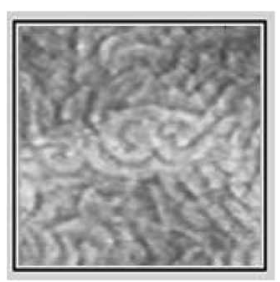

(c)
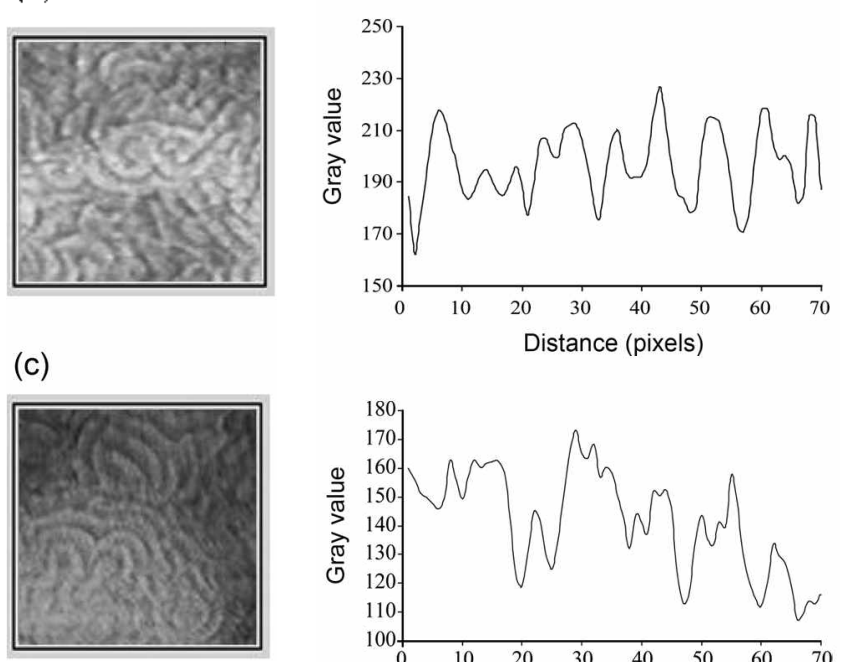

(d)

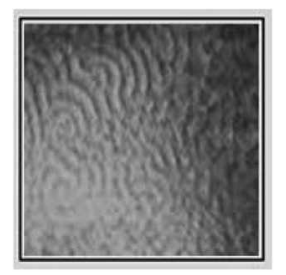

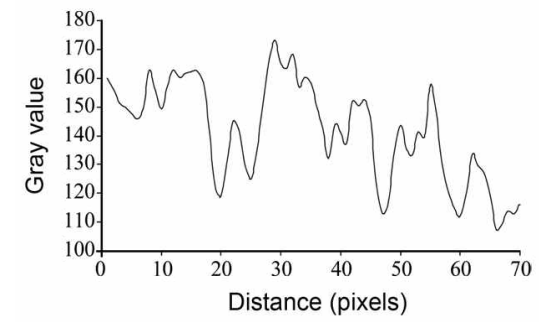

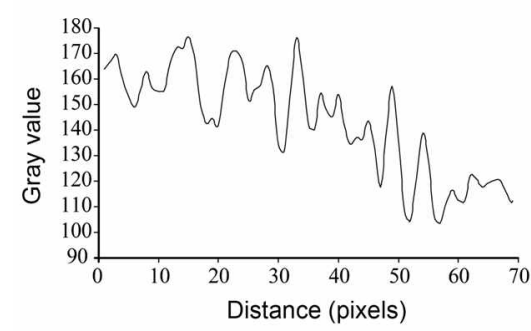

Figure 4. The wave patterns and corresponding plot profiles observed in the system: (a) anti-spiral. (b) chatic, (c) labyrinthine, and (d) Turing-like waves. The spiral pattems are excluded.

cation diagram in the $[1,4-\mathrm{CHD}]_{v}$-[ferroin $]_{v}$ phase. The concentration of [ferroin] $]_{v}$ has been increased from the levels used in Figures 1 and 2 to see in detail the role of a low concentration of [ferroin $]_{v}$ as a metal catalyst for the $\mathrm{BZ}$ reaction system in the variation of pattern change. In this particular instance, the difficulty of obtaining the spiral-type pattern was observed regardless of the propagating direction. Instead, a chaotic type of wave was observed regardless of the $[1,4-\mathrm{CHD}]_{\mathrm{v}}$ levels. Their amplitudes and time periods increased with the level of [1.4-CHD $]_{0}$ used. The increased concentration of [ferroin $]_{v}$ gave rise to more complications in the behavior of the wave patterns, which were identifiable as labyrinthine and hexagonal Turing-like patterns. ${ }^{11}$ These are shown in Figure 4.

The patterns obtained were initiated and propagated throughout the media via the reaction diffusion process in an

excitable media of AOT system. The variation in the initial concentrations in an excitable media provides a lot of possibilities for the variation of the pulse dynamics. The pulse propagation in a medium whose excitation threshold varies periodically in space was studied by considering the $1,4-\mathrm{CHD}-\mathrm{BZ}$ reaction system. However, heterogeneity of the reaction media may also play an important role in the mechanism of pattern formation. Since the reaction medium is a constant state, the waves obtained in this state should be basically dependent on the bifurcation behavior of the reactive intermediates. These intermediates can be controlled by the initial concentrations of the reactant system. In order to obtain a clear view of the bifurcation behavior, a detailed analysis for each pattern is given by the profile diagram similar to Figure 1. Figure 4 shows the profiles of other patterns illustrated in the bifurcation diagrams for particular concentrations for the following wave patterns: (a) spiral wave to inside, (b) chaotic, (c) labyrinthine, and (d) Turinglike. The three patterns except the spiral wave to inside are based on the behavior illustrated in the bifurcation diagrams shown in Figures 2 and 3. These observations clearly indicate the difference of the local dynamics of the excitable media, which are similar to the system with MA as indicated by Vanag. ${ }^{8}$ However, this can behave differently from the MA system since it may be induced by two component species, namely, ferroin and quinone (Q), in which an intermediate of $Q$ arises from the oxidation of 1,4-CHD. These component species are responsible for activating the autocatalysis in the excitation behavior of the 1,4-CHD system. Changes in the concentrations of the reactive intermediate can vary the local dynamics of the reaction. Likewise, these changes can facilitate the recovery of the medium and the onset of a new excitation in the wake of an existing wave. The difference between the conditions for the occurrence of a wave in the $1,4-\mathrm{CHD}$ containing $\mathrm{BZ}$ reaction is a result of the interplay between the two activating species, $1,4 \mathrm{CHD}$ and the quinine-like intermediate. These play an important role in the nature of waves as shown in the bifurcation diagrams of Figures 2 and $3 .^{17}$

Characteristic wave pattern in the 1,4-CHD system. Another observation on the BZ-AOT system containing 1,4$\mathrm{CHD}$ is the induction period. This is the time necessary for the initiation of a wave, and the duration period until the waves disappear when the system changes to an unexcitable condition. Figure 5 illustrates the induction period and life time of wave patterns with respect to the changes in the concentrations of $[1,4-\mathrm{CHD}]_{\mathrm{i}},[\text { Bromate }]_{\mathrm{b}}$, and $[\text { ferroin }]_{0}$ in the BZ-AOT system. The concentrations of the other reagents other than the reagent of variation are the same as in the concentrations illustrated in Figure 1. As the figure shows, the induction periods range from 10 to $50 \mathrm{~min}$. These increased with the increase in the levels of $[1,4-\mathrm{CHD}]_{0}$, but decreased with the increase in the concentrations of $\left[\mathrm{BrO}_{3}{ }^{-}\right]_{0}$ and [ferroin $]_{0}$. These results correspond to the results of Steinbock ${ }^{15}$ and Kurin-Csōrgei et al.$^{18}$ The initiation time for the pattern is shorter compared to other systems carried out either in $\operatorname{resin}^{19}$ or polyacrylamide gel membrane,,$^{20}$ where it 

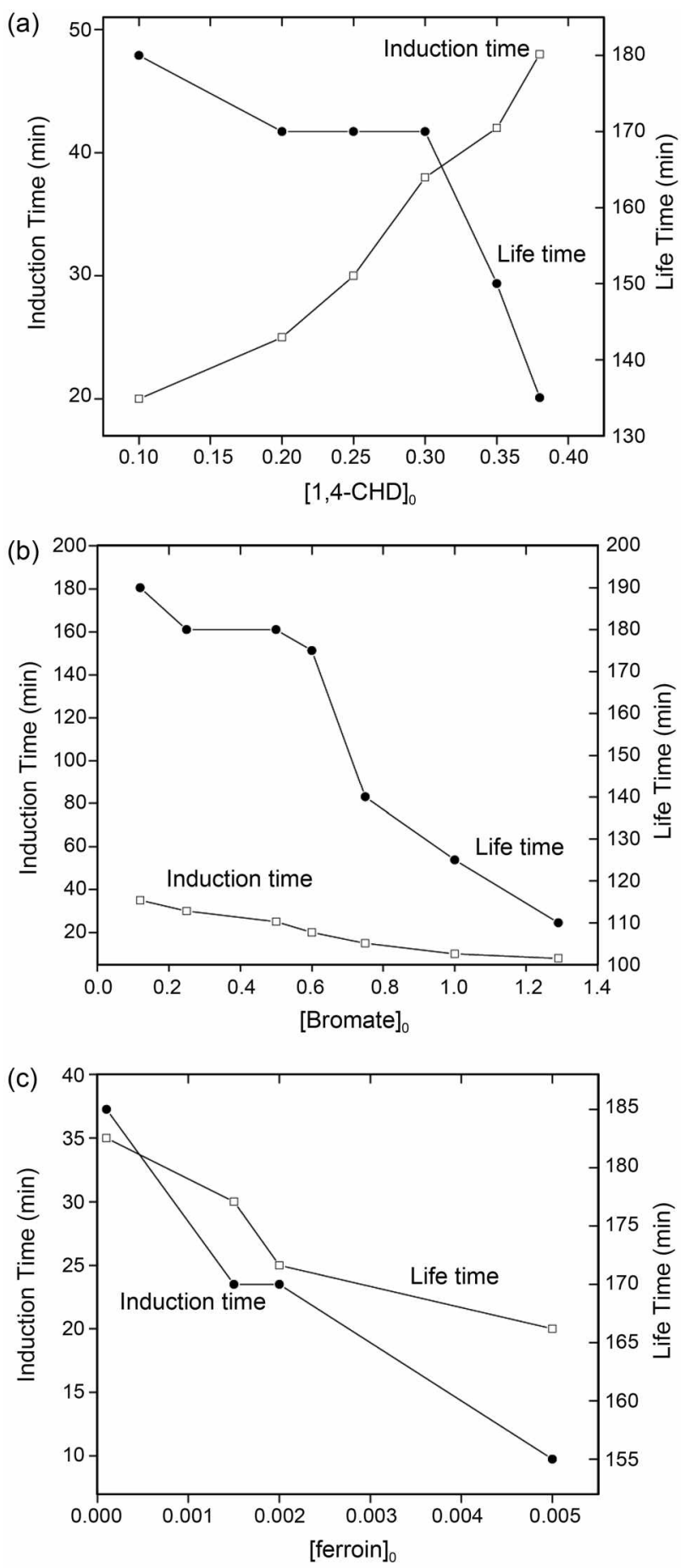

Figure 5. Induction time for the appearance of a pattern and life time duration of the wave pattens in relation to the initial concentrations of (a) [bromate $]_{i}$, (b) $[1,4-\mathrm{CHD}]_{i}$, and (c) [ferroin $]_{0}$. The concentrations of the three reagents in each figure are the same with those indicated in Figure 1.

lies within the $60-80$ and $50-200 \mathrm{~min}$ ranges, respectively. This may be due to the different reaction conditions affecting the mechanical changes in the gel or resin. Solvent diffusivity varies with the media. This factor might have contributed to the observed phase differences between mechanical and chemical oscillations. The shorter induction period in the BZ-AOT system may be explained by the speeding up of the reactant molecules of the $\mathrm{BZ}$ system, which is only observable in the media of micro-emulsions. Diffusion of the reverse micelles and diffusion of the intermediates in the nonpolar phase lead to interesting phenomena in this system. However, the duration of the pattern, that is, the life time, is all inversely proportional to the concentration of $[1,4-$ $\mathrm{CHD}]_{0},\left[\mathrm{BrO}_{3}{ }^{-}\right]_{0}$, and $[\text { ferroin }]_{0}$. This means that the high concentration of the initial reagent brings out the rapid change of the system from an excitable to an unexcitable condition.

The behavior of the 1,4-CHD-bromate-acid reacting system is similar to that of other bromate-driven oscillators. However, the most distinct chemistry of CHD-bromate-acid system is the bromide ion, oxygen transfer, and the one-electron processes occurring alternately. This facet makes the oscillatory system special. Under batch conditions, the bromateCHD reaction shows oscillations or clock behaviors after the induction period. The main processes during the induction period are the bromination of $\mathrm{CHD}$ through its enol form (CHDE), and the formation of hydroquinone $\left(\mathrm{H}_{2} \mathrm{Q}\right)$ through the decomposition of $\mathrm{BrCHD}$;

$$
\begin{gathered}
5 \mathrm{Br}^{-}+\mathrm{BrO}_{3}+6 \mathrm{H}^{-} \rightarrow 3 \mathrm{Br}_{2}+\mathrm{H}_{2} \mathrm{O} \\
\mathrm{CHD}+\mathrm{H}^{-} \rightleftharpoons \mathrm{CHDE}+\mathrm{H}^{+} \\
\mathrm{CHDE}+\mathrm{Br}_{2} \rightarrow \mathrm{BrCHD}+\mathrm{Br}^{-}+\mathrm{H}^{+} \\
\mathrm{BrCHD} \rightarrow \mathrm{H}_{2} \mathrm{Q}+\mathrm{Br}^{-}+\mathrm{H}^{+}
\end{gathered}
$$

Oscillations start when the $\mathrm{H}_{2} \mathrm{Q}$ concentration reaches a critical value, since it plays an important role in the autocatalytic loop:

$$
\begin{aligned}
\mathrm{HBrO}_{2}+\mathrm{BrO}_{3}^{-}+\mathrm{H}^{-} & \rightleftharpoons \mathrm{Br}_{2} \mathrm{O}_{4}+\mathrm{H}_{2} \mathrm{O} \\
\mathrm{Br}_{2} \mathrm{O}_{4} & \rightleftharpoons 2 \mathrm{BrO}_{2} \\
\mathrm{H}_{2} \mathrm{Q}+2 \mathrm{BrO}_{2} & \rightarrow 2 \mathrm{HBrO}_{2}+\mathrm{Q}
\end{aligned}
$$

The explored dynamics by Szalai et al ${ }^{21}$ for the batch oscillators of bromate-CHD reaction indicate that an increase in $\mathrm{Br}^{-}$increases the production rate of $\mathrm{BrCHD}$ and $\mathrm{H}_{2} \mathrm{Q}$. Ferroin affects both positive and negative feedback processes. In the absence of ferroin, along with the above critical concentration of bromide, only clock reaction behavior can be observed. The positive and negative feedback processes of ferroin effect can be explained by the following schemes:

$$
\begin{gathered}
\mathrm{Fe}(\text { phen })_{3}{ }^{2+}+\mathrm{BrO}_{2}+\mathrm{H}^{-} \rightarrow \mathrm{Fe}(\text { phen })_{3}{ }^{3+}+\mathrm{HBrO}_{2} \\
2 \mathrm{Fe}(\text { phen })_{3}{ }^{3-}+\mathrm{BrCHD} \rightarrow \mathrm{Q}+\mathrm{Br}^{-}+3 \mathrm{H}^{+}+2 \mathrm{Fe}(\text { phen })_{3}{ }^{2-}
\end{gathered}
$$

Reaction (R8) competes with (R7), while (R9) produces bromide ions, which act as an inhibitor of the reaction. Low bromide ion concentration results in low $\mathrm{BrCHD}$ concentration. In this case, ferroin reacts with $\mathrm{CHD}$ and produces $\mathrm{H}_{2} \mathrm{Q}$ in (R10):

$$
2 \mathrm{Fe}(\text { phen })_{3}{ }^{3-}+\mathrm{CHD} \rightarrow \mathrm{H}_{2} \mathrm{Q}+2 \mathrm{H}^{-}+2 \mathrm{Fe}(\text { phen })_{3}{ }^{2+}
$$

Owing to (R10), increasing ferroin concentration shortens 
the induction period. The reaction (R9) produces bromide ions, which serve as the inhibitor of this mechanism. At high bromide ion concentration, $\mathrm{BrCHD}$ concentration also becomes relatively high. In effect. (R9) becomes dominant. This is a key step of the BZ-type mechanism.

The main motivation behind this work is to determine the appropriate conditions for studying the dynamics of the BZ system, containing 1,4-CHD system, in an emulsion medium. The short induction period in the AOT system is due to different diffusion coefficients molecules, such as $\mathrm{Br}_{2}$ or $\mathrm{BrO}_{2}$ in oil and dispersed phases. ${ }^{7}$ The degree of interaction among the reacting sites is influenced by the local reaction rate, and the range and strength of the coupling. A change in the local dynamics through interactions refers to the diffusion induced by the chemical turbulence, in which chaos is produced by the local coupling of periodic oscillators. In chemically reacting systems, the local and overall reaction rates can both be influenced by the synchronization of reaction sites.

\section{Conclusions}

This study showed that the dynamics of excitation waves propagating in a periodically modulated AOT-BZ system perform a complex spatio-temporal process. The experimental investigation of the $\mathrm{CHD}-\mathrm{BZ}$ reaction is expected to foster an understanding of the stable pulse propagation in neuronal systems. Depending on the concentrations of the reactants, a characteristic wave evolution was obtained. The most interesting property of excitable media is the existence of autowaves, that is, nonlinear spatio-temporal structures propagating through the medium. In chemical systems, autowave patterns are one of the most evident examples of selforganization in space and time. This finding is interesting not only from a scientific point of view, but also from the new perspective of designing a classical view of the event of neural coding in relation to the rate of discharge. Likewise, this suggests that information is transmitted in precisely timed trains of action potentials. ${ }^{2}$ Since the propagation of excitation pulses in neuronal systems can obey anomalous and even oscillatory dispersion relations, ${ }^{23}$ the resulting stable wave packets could be essential for the reliable transport and encoding of information.

Acknowledgments. This work was supported by the Korea Research Foundation Grant fiunded by the Korean Government (MOEHRD) (KRF-00042007070-00).

\section{References}

1. Kapral, R.; Showalter, K. Chemical Waves and Patterns; Kluwer Academic: Dordrecht, 1995.

2. Cross, M. C.; Hohenberg, P. C. Ret: Mod. Phs. 1993, 65, 851.

3. Belousov, B. P. In Oscillations and Traveling Waves in Chemical Ststens; Field R. J., Burger, M., Eds; Wiley: New York, 1985; Chapter 2.

4. Hamik, C. T.; Manz, N.; Steinbock, O. J. Phis. Chem. A 2001 , 105,6144

5. Muller, S. C. Lectires Notes in Phnsics 1997, 476, 133-148.

6. Manz, N.; Davydov, V. A.; Zykov, V. S.; Muller, S. C. Phis. Rev: E 2002, 66, 036207.

7. Vanag, V. K.; Hanazaki, 1. J. Phis. Chem. 1995, 99, 6944

8. Vanag, V. K. Phys. Uspekh 2004, 47(9), 923.

9. Kaminga, A.; Vanag, V. K.; Epstein, 1. R. J. Chem. Phis. 2005, 122,174706 .

10. Vanag, V. K.; Epstein, I. R. Phys. Ret: Lett. 2004, 92, 128301

11. Vanag, V. K.; Yang, L.; Dolnik, M.; Zhabotinsky, A. M.; Epstein, I. R. Nature 2000, $406,389$.

12. Vanag, V. K.; Zhabotinsky, A. M.; Epstein, I. R. Phis. Rev Lett. $2001,86,552$

13. Turing, A. M. Philos. Trans. R. Soc. London, Ser: B 1952, 237, 37.

14. Vanag, V. K.; Epstein, I. R. Science 2001, 294, 835.

15. Hamik, C. T.; Manz, N.; Steinbock, O. J. Phus. Chem. A 2001, 105,6144 .

16. Manz, M.; Muller, S. C.; Steinbock, O. J. Phis. Chem. A 2000. $104(25), 5897$.

17. Schebesch, I.; Engel, H. Phus. Rer: E 1998, 57(4), 3905.

18. Kurin-Csörgei, K.; Zhabotinsky, A. M.; Orbán, M.; Epstein, I. R. J. Phis. Chem. 1996, 100, 5393 .

19. Huh, D. S.; Choe, S. J.; Kim, M. S. React. Kinet. Catal. Lett. 2001, 74, 11 .

20. Basavaraja, C.; Choe, Y. M.; Hul, D. S. 2008 (to be communicated).

21. Kereszturi, K.; Szalai, I. Chen. Ph:s. Lett. 2006, 428, 288.

22. Berry, M. J.; Warland, D. K.; Meister, M. Proc. Natl. Acad. Sci. US.4 1997, 94, 5411 .

23. Zoccolan, D.; Pinato, G; Torre, V. J. Nenosci. 2002, 23(24), 10790 . 